\title{
IN-BODY RANGING FOR ULTRA-WIDE BAND WIRELESS CAPSULE ENDOSCOPY USING NEURAL NETWORKS BASED ON PARTICLE SWARM OPTIMIZATION
}

\author{
${ }^{1}$ Muzaffer KANAAN, ${ }^{2}$ Rüsstü AKAY, ${ }^{3}$ Memduh SUVEREN \\ 1,2,3 Erciyes University, Department of Mechatronics Engineering, Faculty of Engineering, Kayseri, \\ TURKEY \\ 1'mkanaan@erciyes.edu.tr, 2akay@erciyes.edu.tr, ${ }^{3}$ msuveren@erciyes.edu.tr
}

(Geliş/Received: 20.04.2017; Kabul/Accepted in Revised Form: 11.10.2017)

\begin{abstract}
We consider the problem of accurate in-body ranging for localization of a wireless capsule endoscope utilizing ultra-wide band (UWB) signaling. In this context, we explore the joint use of neural network structures and learning algorithms based on metaheuristics, an example of which is particle swarm optimization (PSO). The contributions of this paper are three-fold. First, we undertake a systematic performance analysis of the PSO technique for UWB-based in-body ranging and propose an improved version of the PSO algorithm. Second, we quantitatively compare the performance of PSO techniques against more traditional learning algorithms, such as Bayesian Regularization, LevenbergMarquardt and Single Conjugate Gradient. Third, we quantify the impact of activation functions used to define the neural network structure on performance. Our results indicate that PSO-based techniques can outperform traditional techniques by as much as $44 \%$, depending on the activation functions used in the neural network.
\end{abstract}

Key Words: In-body ranging, Metaheuristics algorithms, Neural network, Particle swarm optimization, UltraWide band, Wireless capsule endoscopy.

\section{Parçacık Sürü Optimizasyonuna Dayalı Sinir Ağları Kullanılarak Ultra Geniş Bant Kablosuz Kapsül Endoskopide Vücut İçi Mesafe Ölçümü}

ÖZ: Bu makalede, ultra geniş bant (UWB) sinyalleri kullanan bir kablosuz kapsül endoskopunun lokalizasyonu için hassas vücut içi mesafe ölçümü problemi ele alınmaktadır. Bu bağlamda, yapay sinir ağları ve metaheuristik temelli öğrenme algoritmalarının (örnek olarak parçacık sürüsü optimizasyonu (PSO) ortak kullanımı irdelenmektedir. Makalenin literatüre katkıları şu şekilde özetlenebilir. İlk olarak, UWB tabanlı vücut içi mesafe ölçümü için PSO algoritmasının sistematik bir performans analizi yapılmış ve söz konusu problemin çözümü için PSO algoritmasının geliştirilmiş bir versiyonu önerilmiştir. İkinci olarak, önerilen PSO algoritmasının performansı Bayesian Regularization, Levenberg-Marquardt ve Single Conjugate Gradient gibi geleneksel öğrenme algoritmaları ile karşılaştırılmıştır. Son olarak, yapay sinir ağlarında kullanılan aktivasyon fonksiyonlarının performans üzerindeki etkileri incelenmiştir. Elde edilen sonuçlar, önerilen PSO algoritması vasıtası ile geleneksel tekniklere nazaran \% 44'e varan performans artışları elde edilebileceğini göstermektedir.

Anahtar Kelimeler: Vücut içi mesafe ölçümü, Metasezgisel algoritmalar, Sinir ağları, Parçacık sürü optimizasyonu, Ultra geniş bant, Kablosuz kapsül endoskopi.

\section{INTRODUCTION}

Wireless capsule endoscopy (WCE) is a new medical diagnosis technique which, compared to traditional endoscopy techniques, allows the diagnosis of the diseases of the gastrointestinal (GI) tract in 
a relatively painless, non-invasive manner (Swain, 2003). A general overview of the whole system is shown in Figure 1. The camera automatically takes pictures as it goes along the GI tract, and sends the images using an on-board wireless transmitter to a body-mounted receiver. The image data is recorded and can then be reviewed by a gastroenterologist.

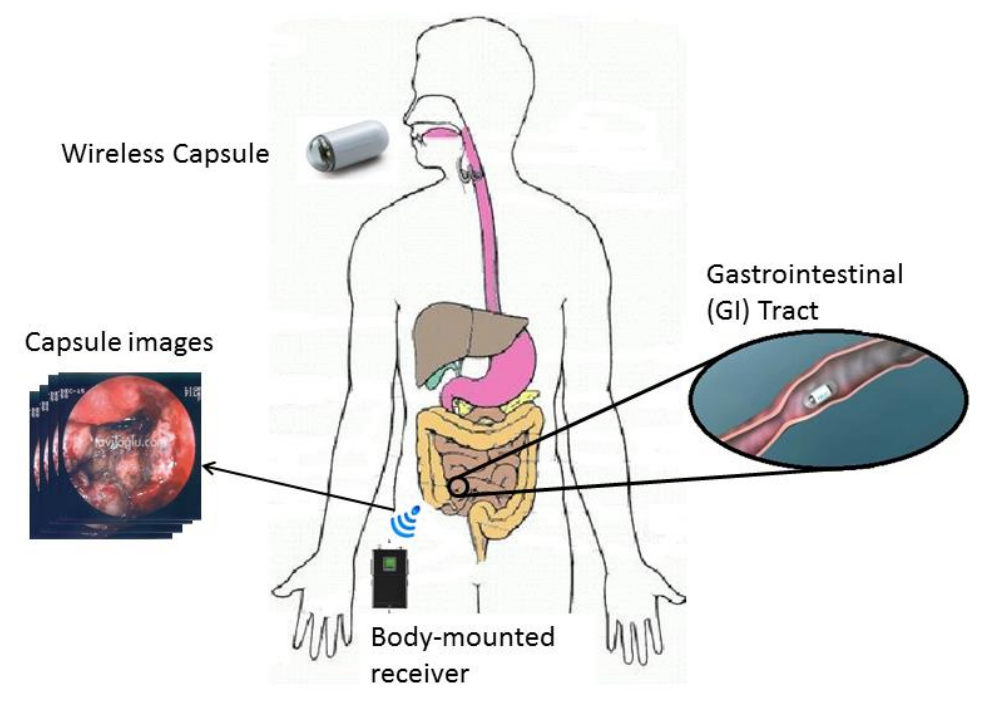

Figure 1. General overview of a wireless capsule endoscopy system

For all of its considerable advantages, current WCE systems also have a deficiency in that it is not possible to correlate the camera images with a unique location in the GI tract. This makes subsequent diagnosis and treatment much more difficult. Therefore, localization of the capsule inside the GI tract needs to be addressed. Localization of the capsule can be performed using range-based techniques (which involve the processing of range, or distance measurements of the wireless transceiver from a number of reference points), angle-based techniques or pattern-recognition techniques (Pahlavan and Levesque, 2005). In this paper our focus is on range-based localization.

For range-based localization to work, it is critical that accurate measurements of the capsule transceiver range from the reference points are available. One of the ways in which this can be accomplished is through the use of ultra-wide band (UWB) technology. Advantages of UWB technology include very high bandwidths (important for high-resolution image transmission from within the GI tract), low-power operation and the ability to accomplish high-accuracy ranging in challenging environments (Yuce et al., 2009; Chavez and Balasingham, 2013). Therefore, we focus on range-based localization using UWB signals in this paper.

However, accurate ranging with UWB signals in the in-body environment is a challenging problem. The human body is an example of a lossy propagation environment, where the electrical permittivity and conductivity of body tissues are frequency-dependent (for an excellent review of this frequency dependence, the interested reader is referred to the work of Gabriel, accessible via http://www.dtic.mil/cgi-bin/GetTRDoc?AD=ADA303903). For UWB-based communication and ranging, characterization of this complicated frequency dependence is of paramount importance and a recent study on this specific topic can be found in (Kanaan and Suveren, 2015). As a result, the UWB signal is distorted in a frequency-selective manner, which makes high-accuracy ranging difficult.

Previous work on in-body ranging using UWB signals can be found in (Chen et al., 2013; Chen, 2013). Traditionally, UWB-based ranging in challenging propagation environments (such as the indoor environment) has been accomplished using the Time of Arrival (TOA) parameter (Lee and Scholtz, 2002; Shang et al., 2013). However, because UWB signals are distorted in a frequency-dependent manner by the body tissues, accurate estimation of the TOA is difficult. Because of this difficulty, it is more feasible to use the strength of the received UWB signal, also known as the Received Signal Strength (RSS) metric, 
for ranging and localization purposes. Prior research has concluded that RSS-based ranging and localization problems can be more effectively solved by means of machine learning techniques, such as neural networks (NNs) (Fang and Lin, 2008; Dai et al., 2016). In this paper, we have chosen to adopt a NN-based approach to the problem of in-body ranging using UWB signals specifically for this reason.

Preliminary studies on NN-based in-body ranging using UWB has been conducted, the results of which have been reported in (Kanaan and Suveren, 2016a; Kanaan and Suveren, 2016b). These works focused on studying the performance of two well-known NN structures, namely the Multilayer Perceptron (MLP) and Cascade MLP (C-MLP) structures, in the presence of the Levenberg-Marquardt (L-M), Bayesian Regularization (BR) and Single Conjugate Gradient (SCG) training algorithms and varying number of neurons in the hidden layer. A considerably expanded study that compared the performance of NN-based range estimators versus other estimators (such as those that are based on the CLEAN algorithm) is presented in (Kanaan and Suveren, 2017).

Since the process of training a neural network can be framed as an optimization problem, the following question deserves to be answered: are there other optimization approaches that could enhance the performance of NN-based ranging? A possible answer to this question may come in the form of metaheuristics, which take inspiration from biological phenomena, such as the behavior of schools of fish, ant colonies, in order to solve challenging optimization problems (Yang, 2008; Talbi, 2009). Our main objective in this paper is to perform a more extensive investigation on NN-based in-body ranging using UWB signals, where the NN is trained with metaheuristics techniques. A literature review of various metaheuristics algorithms used for training NN are available in (Alba and Marti, 2006; Boussaïda et al., 2013). Due to space limitations, we have arbitrarily chosen to focus on only one such technique, namely, particle swarm optimization (PSO) (Kennedy and Eberhart, 1995; Ch and Mathur, 2012; Garro and Vázquez, 2015; Garg et al., 2014).

As the first contribution of this paper, we present a systematic performance analysis of an NN-based range estimator trained using the PSO algorithm, and propose an improved PSO algorithm using the strategy of elitism. The second contribution of this paper is to quantitatively compare the performance of NN-based range estimators where the NN is trained in accordance with the PSO algorithm, versus the cases where the NN is trained with more traditional algorithms, such as the Levenberg-Marquardt (LM), Bayesian Regularization (BR) and Single Conjugate Gradient (SCG) algorithms. Our results indicate that the use of metaheuristics techniques such as PSO can noticeably improve the performance of NNbased range estimators, as opposed to standard NN training algorithms such as LM, BR and SCG. The third and final contribution of the paper is to quantify the impact of different activation functions used for individual neurons in the network on the final ranging performance. It should be emphasized at this juncture that our objective in this paper is simply to explore the application of PSO techniques specifically to the solution of the NN-based in-body ranging problem.

The rest of this paper is organized as follows. In section 2, we present details of the simulation and performance analysis environment used for this study. The use of the PSO algorithm for in-body ranging is presented in section 3. In section 4, performance results and a quantitative discussion of those results are given. Section 5 concludes the paper.

\section{SIMULATION AND PERFORMANCE ANALYSIS ENVIRONMENT}

The first phase of our study on UWB-based in-body ranging involves the characterization of the propagation environment in this environment for the specified band. For this purpose, we have employed the use of a 3-D electromagnetic simulation software, known as XFDTD ${ }^{\mathrm{TM}}$, from Remcom, Inc. This package allows the calculation of electric and magnetic field intensities over a region of interest, using the Finite Difference Time Domain (FDTD) technique, subject to boundary conditions specified in the problem (for more information on this software package, the interested reader is referred to http://www.remcom.com/xf7). Since we are interested in characterizing the in-body environment for this study, a 3-D voxel model of the human body is required. The model utilized for this study was based on 
the Visible Human dataset, developed and maintained by the United States National Library of Medicine (for more details of this data set, please see http://www.nlm.nih.gov/research/visible/visible human.html). Figure 2-a shows a general view of the whole-body 3-D voxel model, which has been truncated in order to keep calculation times within tolerable limits, as illustrated by Figure 2-b.

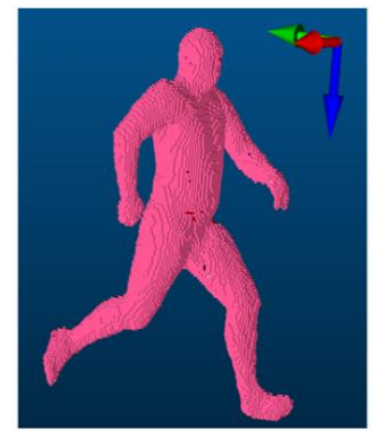

(a)

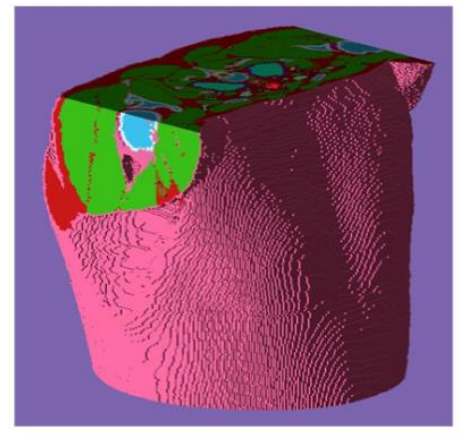

(b)

Figure 2. 3-D voxel models used for performance analysis (a) the whole-body model

(b) truncated chest model.

Parameter values used in the FDTD calculation were as follows. FDTD cell size was $2 \mathrm{~mm}$ in the $\mathrm{x}$ and y directions, and $0.5 \mathrm{~mm}$; these values were selected in order to satisfy the Courant limit. The Perfectly Matched Layer (PML) boundary condition was applied to the 3-D model in order to account for the effects of reflections from the body environment.

Since the scenario focused on in this study was UWB-based WCE, the next step was to embed a UWB transmit antenna (as a proxy for the actual endoscopy capsule) within the GI tract. Since WCE is mostly used to image the small intestine, the UWB antenna was inserted into the body model at several locations within the small intestine, in order to model the UWB signal characteristics there. A total of 32 locations within the small intestine were selected, as depicted in Figure 3-a and Figure 3-b. The particular antenna used for this study was the elliptic disc dipole antenna, illustrated in Figure 3-c, details of which are given in (Khaleghi and Balasingham, 2009). It should be noted, however, that the overall size of the antenna was scaled down by a factor of two, in order to make the antenna small enough to fit in the small intestine. The antenna was fed with a modulated Gaussian pulse, with a center frequency of $4.1 \mathrm{GHz}$ and a $-10 \mathrm{~dB}$ bandwidth of $1.4 \mathrm{GHz}$. In this manner, we have ensured that most of the spectral content of the transmitted signal resided in the $3.4-4.8 \mathrm{GHz}$ range, which has come to be known as the "UWB low band" in the literature. The rationale for this choice was the fact that this part of the UWB band has more favorable propagation characteristics in the in-body environment (Wang and Wang, 2013). For the receive side, an array of receive antennas, modeled as point sensors, were incorporated into the simulation model. The number of these point sensors was fixed at 64 . 


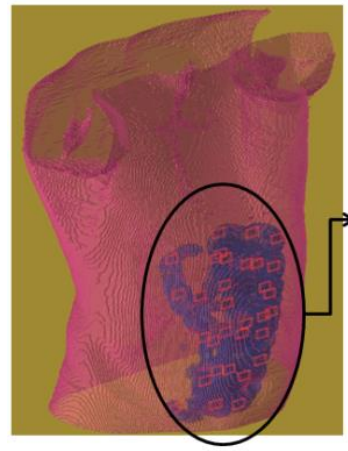

(a)

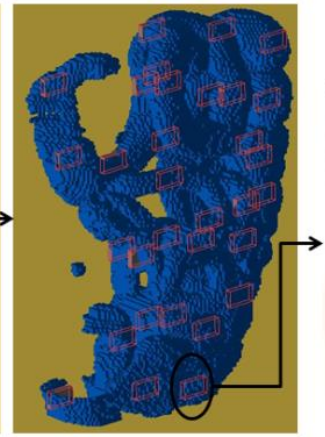

(b)

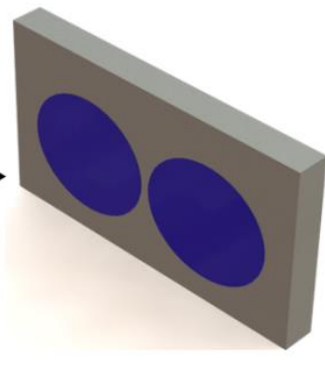

(c)

Figure 3. Antenna locations within the small intestine: (a) General view (b) Detail view (c) Elliptic disc dipole antenna

Electric and magnetic field intensity values obtained in this manner were used to calculate values of the Poynting vector, $\mathbf{P}(t)$, defined as the cross product

$$
\mathbf{P}(t)=\mathbf{E}(t) \times \mathbf{H}(t)
$$

where $\mathbf{E}(t)$ and $\mathbf{H}(t)$ are the values of the time-dependent electric and magnetic field intensities as calculated by the FDTD tool, respectively. The magnitude of $\mathbf{P}(t)$ at each of the receive sensors was calculated for every possible location of the transmit antenna within the small intestine; this makes for a total of $32 \times 64=2048$ data sets. The Fast Fourier Transform (FFT) of each of these data sets was computed, and the resulting data set was down sampled at $40-\mathrm{MHz}$ intervals in order to reduce the size of the data set. The training time for the NN and the memory requirements are both affected by the size of the input data set; therefore, this down sampling step was found to be necessary in order to keep these parameters within reasonable limits. This reduced dataset was then used to train a neural network. The process of training the network, i.e. the method through which the synaptic weights were adjusted, was accomplished using the PSO (discussed in greater detail in section 3), as well as the BR, LM and SCG algorithms, and two different activation functions, namely the log-sigmoid (logsig) and tangent-sigmoid (tansig) functions.

\section{NN-BASED RANGING WITH THE PSO ALGORITHM}

In this section, we first briefly review the basic PSO algorithm. The PSO algorithm, which was introduced by Kennedy and Eberhart in 1995, is an optimization algorithm that is inspired by the movement of organisms in a bird flock or fish school (Kennedy and Eberhart, 1995). In the basic PSO, the population is considered as a swarm and each particle in the population is considered a potential solution. The algorithm updates these particle positions in the search space to find the optimal solution. The structure of the basic PSO algorithm is as given in Algorithm 1. below.

Algorithm 1. The main steps of the PSO algorithm

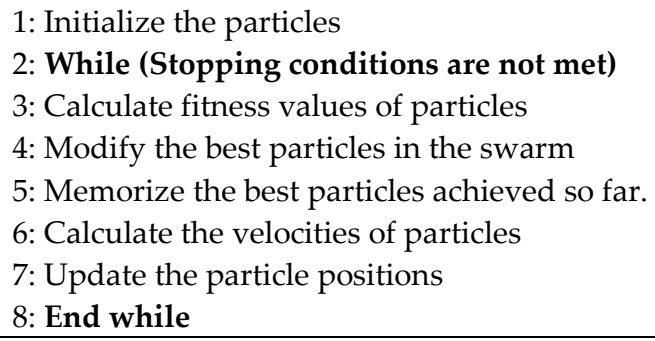


The algorithm determines the modifications to particle velocities by using the personal best and global best values. The modification of particle velocities and positions are obtained by using the relations

$$
\begin{aligned}
& x_{i}(t+1)=x_{i}(t)+v_{i}(t+1) \\
& v_{i}(t+1)=w v_{i}(t)+c_{1} k_{1}\left(x_{i}^{\text {best }}-x_{i}(t)\right)+c_{2} k_{2}\left(x^{\text {gbest }}-x_{i}(t)\right)
\end{aligned}
$$

where $x_{i}(t)$ is the position of the $i$ th particle at time $t$ and $v_{i}(t+1)$ is the velocity for the $i$ th particle at time $t+1$. $x_{i}^{\text {best }}$ represents the personal best solution for particle $i$ and $x^{\text {gbest }}$ represents global best positions over all particles. $w$ is the inertia weight which determines how the previous velocity of the particle influences the velocity in the next iteration. $c_{1}$ and $c_{2}$ are learning factors whose values should be between 0 and 4 . The parameters $k_{1}$ and $k_{2}$ are two random numbers drawn from a uniform random number generator in the interval [0,1]. These parameters can have a large impact on optimization performance. Selection of the optimum values for all these parameters has been the subject of much research (Shi and Eberhart, 1999; Uy et al., 2007; Xin et al., 2009; Liu et al., 2010; Jordehi and Jashi, 2013).

The aim of all metaheuristics techniques is to iteratively improve the quality of the population. For this purpose almost all evolutionary algorithms use greedy selection which means selecting the better one between the current solution and new candidate solution, while the PSO algorithm replaces the old solution with the candidate solution directly without checking performance. This creates the risk that the best solutions in the population might be lost. In order to avoid deterioration of the current solution, most evolutionary algorithms utilize the concept of elitism.

In order to improve the performance of the basic PSO algorithm, we propose a modification, known as Improved PSO (IPSO). IPSO utilizes the strategy of elitism where the worst solutions are replaced by a certain number of the best solutions in every generation. In other words, at every iteration, a certain number of best-performing solutions are carried forward to the next iteration. We formalize this replacement by defining a "keep- $x$ " parameter, where $x$ represents the number of best-performing solutions that are carried forward to the next iteration; the lower the value of $x$, the more elitist the algorithm will be. If this replacement results in duplicate solutions, they are modified randomly to continue the search beyond the local optimal solution as detailed in Algorithm 2 below.

Algorithm 2. The main steps of the IPSO algorithm
1: Initialize the particles
2: While (Stopping conditions are not met)
3: Calculate fitness values of particles
4: Modify the best particles in the swarm
5: Memorize the best particles achieved so far.
6: Calculate the velocities of particles
7: Update the particle positions
8: Replace $\mathrm{x}$ worst solutions with elite solutions
9: Modify duplicate solutions
10: End while

\section{RESULTS AND DISCUSSION}

The performance of PSO, IPSO, BR, LM and SCG algorithms was evaluated under different NN configurations and different activation functions. For all cases, the NN structure involved one input layer, one output layer and a single hidden layer. The number of neurons in the hidden layer was varied between 4 and 32. The activation functions used on the individual neurons were varied between the tangent-sigmoid and log-sigmoid functions. For the sake of simplicity, the same activation function was 
used on all neurons in the network. For the PSO and IPSO algorithms, the population size was 64, the maximum number of cycle was 1000, the learning factors were both set to 1.8 and inertia weight was set to 0.6 as recommended in (Vesterstrom and Thomsen, 2004). All algorithms were run 30 times for a statistically meaningful comparison of the results. In addition, the data sets were normalized to the range $[0,1]$ and randomly shuffled before training the network.

The first set of results we present involves a comparison of the standard PSO and IPSO algorithms for different hidden layer sizes, and the use both of the log-sigmoid (logsig) and tangent-sigmoid (tansig) activation functions. These results are given in Table 1. For the case of the IPSO algorithm, the impact of the degree of elitism was also investigated, where a certain number of best-performing solutions are kept between one iteration of the algorithm to the next: the scenario 'IPSO-Logsig-Keep 4', for example, means that the four best-performing solutions are carried forward to the next iteration (i.e. the value of $x$ in the "keep- $x$ " parameter equals 4 ). In order to come up with a single figure of merit to compare all scenarios, the average root mean square error (RMSE) across all hidden layer sizes is also computed and presented in Table 1, and the algorithms are ranked on the basis of best-case performance for each type of activation function. Note that in the tables, the scenarios with the best individual performance are highlighted in bold type.

Table 1. Comparison of the ranging RMSE (in $\mathrm{mm}$ ) between IPSO and standard PSO for different hidden layer sizes and activation functions.

\begin{tabular}{|c|c|c|c|c|c|c|c|}
\hline \multirow{2}{*}{$\begin{array}{l}\text { Activation } \\
\text { Function }\end{array}$} & \multirow{2}{*}{$\begin{array}{l}\text { Simulation } \\
\text { Scenario }\end{array}$} & \multicolumn{4}{|c|}{ Number of neurons in the hidden layer } & \multirow{2}{*}{$\begin{array}{l}\text { Mean } \\
\text { RMSE }\end{array}$} & \multirow{2}{*}{$\begin{array}{c}\text { Rank of } \\
\text { Mean } \\
\text { RMSE }\end{array}$} \\
\hline & & 4 & 8 & 16 & 32 & & \\
\hline \multirow{5}{*}{ Logsig } & IPSO-Keep 32 & 31.44 & 30.41 & 30.29 & 29.88 & 30.50 & 5 \\
\hline & IPSO-Keep 16 & 30.86 & 29.91 & 29.84 & 29.78 & 30.10 & 1 \\
\hline & IPSO-Keep 8 & 30.04 & 29.85 & 30.43 & 30.18 & 30.13 & 2 \\
\hline & IPSO-Keep 4 & 30.99 & 29.98 & 29.97 & 30.02 & 30.24 & 4 \\
\hline & PSO & 30.42 & 30.55 & 29.82 & 29.83 & 30.16 & 3 \\
\hline \multirow{5}{*}{ Tansig } & IPSO-Keep 32 & 29.52 & 29.59 & 29.66 & 29.28 & 29.51 & 3 \\
\hline & IPSO-Keep 16 & 29.35 & 29.34 & 29.22 & 29.13 & 29.26 & 1 \\
\hline & IPSO-Keep 8 & 29.63 & 29.26 & 29.41 & 29.48 & 29.44 & 2 \\
\hline & IPSO-Keep 4 & 30.30 & 29.33 & 29.68 & 29.64 & 29.74 & 4 \\
\hline & PSO & 29.69 & 29.65 & 30.18 & 30.38 & 29.98 & 5 \\
\hline
\end{tabular}

One striking observation we can make on the basis of Table 1 is that the performance of IPSO does not necessarily get better as the algorithm becomes more elitist, i.e. as the value of $\mathrm{x}$ in the "keep- $\mathrm{x}$ " parameter is lowered. This is readily seen from the first four rows of Table 1, where we see that a value of $x=4$ does not lead to the best ranging performance across all hidden layer sizes. In fact, the best average performance across all hidden layer sizes is attained when $x=16$. The results of Table 1 , the best-performing IPSO scenario has a better performance compared to the PSO algorithm for both logsig and tansig transfer functions. This somewhat counterintuitive finding may have to do with the size of the data set used to train the network, as well as a host of other nonlinear effects that are characteristic of a computational structure such as a neural network, and deserves further investigation.

The next set of results involves a comparison of the BR, LM, SCG and IPSO algorithms on their own based on the logsig and tansig activation functions, as presented in Table 2. 
Table 2. Comparison of the ranging RMSE (in mm) between BR, LM, SCG and IPSO algorithms for different hidden layer sizes and activation functions.

\begin{tabular}{|c|c|c|c|c|c|c|c|}
\hline \multirow{2}{*}{$\begin{array}{l}\text { Activation } \\
\text { Function }\end{array}$} & \multirow{2}{*}{$\begin{array}{l}\text { Simulation } \\
\text { scenario }\end{array}$} & \multicolumn{4}{|c|}{ Number of neurons in the hidden layer } & \multirow{2}{*}{$\begin{array}{l}\text { Mean } \\
\text { RMSE }\end{array}$} & \multirow{2}{*}{$\begin{array}{c}\text { Rank of } \\
\text { Mean } \\
\text { RMSE }\end{array}$} \\
\hline & & 4 & 8 & 16 & 32 & & \\
\hline \multirow{4}{*}{ Logsig } & $\mathrm{BR}$ & 53.61 & 53.40 & 53.67 & 53.02 & 53.42 & 3 \\
\hline & LM & 54.47 & 53.70 & 54.65 & 54.79 & 54.40 & 4 \\
\hline & SCG & 53.65 & 53.16 & 53.06 & 53.07 & 53.24 & 2 \\
\hline & IPSO & 30.86 & 29.91 & 29.84 & 29.78 & 30.10 & 1 \\
\hline \multirow{4}{*}{ Tansig } & $\mathrm{BR}$ & 29.95 & 30.32 & 32.25 & 31.19 & 30.93 & 3 \\
\hline & $\mathrm{LM}$ & 31.96 & 36.45 & 32.33 & 37.72 & 34.61 & 4 \\
\hline & SCG & 29.96 & 29.67 & 29.59 & 29.33 & 29.64 & 2 \\
\hline & IPSO & 29.35 & 29.34 & 29.22 & 29.13 & 29.26 & 1 \\
\hline
\end{tabular}

We can immediately infer from Table 2 that, on average, the best performing algorithm across all hidden layer sizes is the IPSO algorithm. In the case of the logsig activation function, we see that IPSO enjoys a clear performance advantage against BR, LM and SCG algorithms. The exact amount of this performance can be found by comparing the average RMSE figures and is seen to be approximately in the $43.5-44.5 \%$ range. In the case of the tansig activation function, however, such a big performance gap does not seem to exist; the results indicate that amount of performance improvement obtained by using IPSO is approximately in the $1.3-5 \%$ range. The point to take away from these results is that the IPSO algorithm, and thus metaheuristics techniques in general, do have potential for accurate in-body ranging. However, the performance improvement obtained by the IPSO algorithm is dependent on the activation function used.

For a different perspective on these results, we now turn to Figure 4, where, for the case of the logsigmoid activation function, the best-performing IPSO scenario (referred to as "IPSO-logsig-keep 16" with 32 neurons in Table 1) is compared against the traditional algorithm with the best individual performance, namely the BR algorithm with 32 neurons. The comparison is now on the basis of the cumulative distribution function of the ranging error obtained empirically from the simulation results. The figure reveals that the IPSO algorithm attains a ranging error of $48.3 \mathrm{~mm}$ or less with $90 \%$ probability, whereas the BR algorithm is able to achieve a ranging error of $87.94 \mathrm{~mm}$ or less with the same probability. For the tangent-sigmoid activation function, the IPSO algorithm is compared in the same manner against the best-performing traditional algorithm, which turns out to be the SCG algorithm. These results are shown in Figure 5, where we see that both algorithms are able to attain a ranging error of approximately $50 \mathrm{~mm}$ or less with $90 \%$ probability. The main point here is that the IPSO algorithm is able to achieve a significant performance advantage against traditional algorithms, although this performance advantage is also dependent on the choice of the activation function. For yet another perspective on the results of Figure 4 and Figure 5, the algorithms exhibiting the best performance for the different activation functions are compared on the basis of the mean average error (MAE) and the mean relative error (MRE) metrics, as seen in Table 3. 


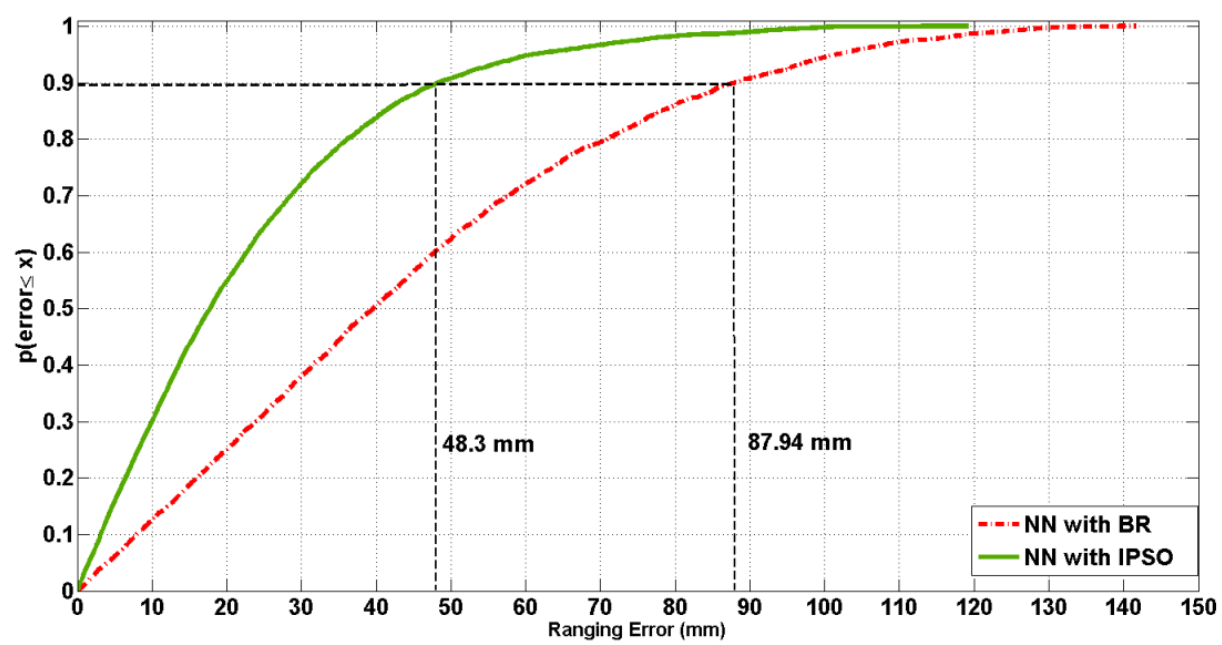

Figure 4. Statistical comparison of the IPSO algorithm versus the best-performing traditional algorithm (BR): Log-sigmoid activation function.

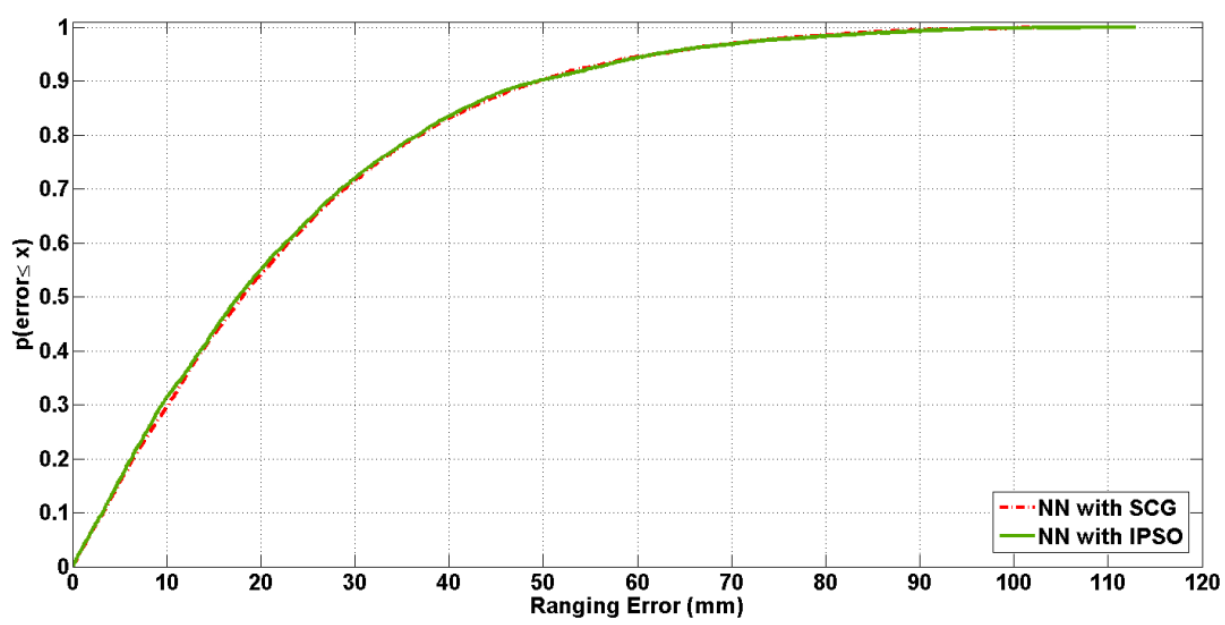

Figure 5. Statistical comparison of the IPSO algorithm versus the best-performing traditional algorithm (SCG): Tangent-sigmoid activation function.

Table 3. Comparison of the RMSE, MAE, MRE values between BR, SCG and IPSO algorithms

\begin{tabular}{|c|cccc|}
\hline $\begin{array}{c}\text { Activation } \\
\text { Function }\end{array}$ & Algorithm & RMSE & MAE & MRE \\
\hline \multirow{2}{*}{ Logsig } & BR & 53,02 & 44,11 & $39,87 \%$ \\
& IPSO & 29,78 & 22,69 & $15,41 \%$ \\
\multirow{2}{*}{ Tansig } & SCG & 29,33 & 22,91 & $15,42 \%$ \\
& IPSO & 29,13 & 22,69 & $15,39 \%$ \\
\hline
\end{tabular}

It may be instructive to compare the results given above with other studies in the literature dealing with the problem of NN-based in-body ranging. Unfortunately, in the course of our literature search we were unable to locate any other studies in the open literature dealing with this specific problem that would permit a direct comparison.

The results given above serve to highlight two critically important points with regards to the problem of in-body ranging using UWB signals. The first is that metaheuristics techniques such as PSO 
may have significant potential in terms of accomplishing accurate UWB-based in-body ranging. The performance advantage of PSO techniques and the IPSO algorithm in particular, can clearly be seen even with other performance metrics such as the MAE and the MRE, as seen in Table 3. The second point is that it is not just the training algorithm, but also the choice of the activation function, which significantly impacts performance. These results serve to advance the state of the art which will hopefully make accurate in-body ranging and localization a reality in the near future.

\section{CONCLUSIONS}

In this paper, we discussed the problem of accurate in-body ranging using UWB signaling and explored the utility of metaheuristics concepts, such as PSO, for accomplishing accurate UWB-based inbody ranging through NN structures. Our results clearly indicate the potential of such techniques for this problem, with PSO sometimes outperforming more traditional learning algorithms such as BR, LM and SCG by as much as $44 \%$ depending on the activation function used in the NN structure. The results help to advance the state of the knowledge in the area of accurate UWB-based in-body ranging.

\section{ACKNOWLEDGMENT}

The authors would like to thank the anonymous reviewers in advance for their time and thoughtful comments. This work was supported by the Erciyes University Research Projects Fund under grant number FYL-2014-5429. The authors declare that they have no conflicts of interest.

\section{REFERENCES}

Alba, E., Marti, R., 2006, Metaheuristic Procedures for Training Neural Networks, Operations Research/Computer Science Interfaces Series, Springer, New York, NY, USA.

Boussaïda, I., Lepagnot, J., Siarry, P., 2013, "A Survey on Optimization Metaheuristics", Information Sciences, Vol. 237, pp. 82-117.

Ch, S., Mathur S., 2012, "Particle Swarm Optimization Trained Neural Network for Aquifer Parameter Estimation", KSCE Journal of Civil Engineering, Vol. 16, No. 3, pp. 298-307.

Chavez-Santiago R., Balasingham I., "Computation of the Transmission Frequency Band for The Ultra Wideband Capsule Endoscope", 7th International Symposium on Medical Information and Communication Technology (ISMICT), Tokyo, Japan, pp. 66-70, 6-8 March 2013.

Chen, J., 2013, UWB Characteristics of RF Propagation for Body Mounted and Implanted Sensors, MSc Thesis, Worcester Polytechnic Institute, MA.

Chen, J., Ye, Y., Pahlavan, K., "Comparison of UWB and NB RF Ranging Measurements in Homogenous Tissue for BAN Applications", Wireless Telecommunications Symposium (WTS), Phoenix, Arizona, USA, pp.1-5, 17-19 April 2013.

Dai, H., Ying, W. H., Xu, J., 2016, “Multi-layer Neural Network for Received Signal Strength-Based Indoor Localisation", IET Communications, Vol. 10, No. 6, pp. 717-723.

Fang, S. H., Lin, T. N., 2008, “Indoor Location System Based on Discriminant-Adaptive Neural Network in IEEE 802.11 Environments", IEEE Transactions on Neural Networks, Vol. 19, No. 11, pp.19731978.

Garg, S., Patra, K., Pal, S. K., 2014, "Particle Swarm Optimization of a Neural Network Model in a Machining Process", Sadhana, Vol. 39, No. 3, pp. 533-548.

Garro, B. A., Vázquez, B. A., 2015, "Designing Artificial Neural Networks Using Particle Swarm Optimization Algorithms", Computational Intelligence and Neuroscience, pp. 1-20, http://dx.doi.org/10.1155/2015/369298

Jordehi, A. R., Jashi, J., 2013, "Parameter Selection in Particle Swarm Optimisation: a Survey", Journal of Experimental \& Theoretical Artificial Intelligence, Vol. 25, No. 4, pp.527-542. 
Kanaan, M., Suveren, M., “In-body Ranging for Ultra-Wide Band Wireless Capsule Endoscopy Using a Neural Network Architecture", 10 ${ }^{\text {th }}$ International Symposium on Medical Information and Communication Technology (ISMICT), Worcester, USA, 1-5, 20-23 March 2016 a.

Kanaan, M., Suveren, M., "Ranging for In-Body Localization of Ultra Wide Band Wireless Endoscopy Capsules using Neural Networks", 24th Signal Processing and Communication Application Conference, (SIU-2016), Zonguldak, Turkey, 16-19 May, 2016b.

Kanaan, M., Suveren, M., "In-Body Ranging with Ultra-Wideband Signals: Techniques and Modeling of the Ranging Error", 2017, Wireless Communications and Mobile Computing, 2017, pp. 1-15.

Kanaan, M., Suveren, M., 2015, “A Novel Frequency-Dependent Path Loss Model for Ultra Wideband Implant Body Area Networks", Measurement, Vol. 68, pp. 117-127.

Kennedy, J., Eberhart, R., "Particle Swarm Optimisation", IEEE International Conference on Neural Networks, WA, Australia, pp. 1942-1948, 27-1 December 1995.

Khaleghi, A., Balasingham, I., 2009, “Improving in-Body Ultra Wideband Communication Using NearField Coupling of the Implanted Antenna", Microwave and Optical Technology Letters, Vol. 51, pp. 585-589.

Lee, J. Y., Scholtz, R. A, 2002, "Ranging in a Dense Multipath Environment Using an UWB Radio Link", IEEE Journal on Selected Areas in Communications, Vol. 20, No.9, pp.1677-1683, DOI:10.1109/JSAC.2002.805060.

Liu, C., Ouyang, C., Zhu, P., Tang, W., "An Adaptive Fuzzy Weight PSO Algorithm", 2010 Fourth International Conference on Genetic and Evolutionary Computing, Shenzhen, China, pp. 8-10, 13-15 December 2010.

Pahlavan, K., Levesque, A. H., 2005, Wireless Information Networks, 2nd Edition, John Wiley \& Sons, Inc., Hoboken, New Jersey, USA.

Shang, F., Champagne, B., Psaromiligkos, I., 2013, “Time of Arrival and Power Delay Profile Estimation for IR-UWB Systems", Signal Processing, Vol. 93, pp.1317-1327, DOI: 10.1016/j.sigpro.2012.11.006.

Shi, Y., Eberhart, R., "Empirical Study of Particle Swarm Optimisation", IEEE International Conference on Computational Intelligence, Washington, USA, 1945-1950, 6-9 July 1999.

Swain P., 2003, “Wireless capsule endoscopy", Gut, Vol.52, p.iv48-iv50.

Talbi, E., 2009, Metaheuristics: from Design to Implementation, John Wiley \& Sons, Hoboken.

Uy, N. Q., Hoai, N. X., Mckay, R., Tuan, P. M., "Initialising PSO with Randomised Lowdiscrepancy Sequences: The Comparative Results", IEEE Congress on Evolutionary Computation, Singapore, pp. 1985-1992, 25-28 September 2007.

Vesterstrom, J., Thomsen, R., "A Comparative Study of Differential Evolution, Particle Swarm Optimization, and Evolutionary Algorithms on Numerical Benchmark Problems", Congress on Evolutionary Computation, Porrtland, pp. 1980-1987, 19-23 June 2004.

Wang, J., Wang, Q., 2013, Body Area Communications: Channel Modeling, Communication Systems and EMC, John Wiley \& Sons, Singapore.

Xin, J., Chen, G., Hai, Y., "A Particle Swarm Optimiser with Multi-Stage Linearly Decreasing Inertia Weight", In IEEE International Conference on Computational Sciences and Optimisation, Sanya,China, pp. 505-508, 24-26 April 2009.

Yang, X. S., 2008, Nature-inspired Metaheuristic Algorithms, Luniver Press, UK.

Yuce, M. R., Keong, H. C, Chae, M. S, 2009, “Wideband Communication for Implantable and Wearable Systems", IEEE Transactions on Microwave Theory and Techniques, Vol. 57, No. 10, pp.2597-2604. 